\title{
Stability, Stabilization and Experiments for Networked Control Systems with Random Time Delay
}

\author{
Chih-Chung Chen, Sandra Hirche and Martin Buss
}

\begin{abstract}
It is well known that the stability and performance of a networked control system (NCS) are strongly affected by the transmission delay, which is usually random in communication networks such as Ethernet. In order to cope with the random transmission delay and increase the control performance, a novel switching control approach for NCS is proposed. The random transmission delay is modelled by a Markov process. A controller, which is able to monitor the transmission delay and synchronously switches with the delay, is considered. The resulting closed-loop system is a Markovian jump linear system (MJLS) with random delay. In this paper a delay-dependent stability condition for stochastic exponential mean square stability is derived by using a LyapunovKrasovskii functional. The controller design algorithm for a switching controller is proposed. Experiments with the 3 degree-of-freedom (DoF) robotic manipulator ViSHaRD3 ${ }^{1}$ show the validity of the proposed approach. An alternative nonswitching approach with buffering strategy at the controller side is considered. The experimental comparison with the nonswitching counterpart indicates performance benefits for the proposed switching control approach.
\end{abstract}

\section{INTRODUCTION}

A networked control system (NCS) is a feedback control system using a shared network for the communication between spatially distributed sensors, actuators and physical plants. The NCS has advantages such as low cost, high flexibility, easy installation and maintenance, which facilitate its applications in automation technology. Typical examples are unmanned aerial vehicles [1], Ethernet-based car control network [2] and teleoperation [3].

The use of a communication network comes, however, at the price of non-ideal signal transmission: the sampled data sent through the network experience variable time delays and suffer transmission losses (or packet dropouts), see [4]. Particularly, the delay is well known as a source of instability and deteriorates the control performance [5]. So far, various approaches have been proposed in the literature to cope with the delay, see [6]-[10]. In [6], the augmented state vector method for constant delay is proposed. A hybrid system analysis approach is applied to NCS in [7] for known delay and in [8] for uncertain delay. Time-varying delay and robust control are addressed in [9]. In [10], a delay compensation predictive control approach is proposed for the delay with known deviations. More approaches with deterministic delays can be found in [11], [12] and references therein.

\footnotetext{
Institute of Automatic Control Engineering, Technische Universität München, D-80290 München, Germany; http://www.lsr.ei.tum.de, \{s.hirche, m.buss\}eieee.org

${ }^{1}$ Virtual Scenario Haptic Rendering Device
}

Systems with random time delay are studied in [13]-[17]. In [13], the delay is modelled as a Markov process and the effect of random delay is treated as an LQG problem. However, the network-induced random delay has to be less than one sampling interval. Therefore, this approach may be unsuitable for systems with longer time delay. A stochastic hybrid system approach involving bounded random delay and switching feedback control laws is considered in [14]. The approach results in a bilinear matrix inequality (BMI). An iteration algorithm is formulated for solving the BMI difficulties. The model-based NCS with random transmission delay is studied in [15]. Sufficient conditions for almost sure stability and stochastic exponential mean square stability are presented. In [16], a $H_{\infty}$ control problem for Bernoulli binary random delay is considered and an LMI problem for the analysis of stochastic exponential mean square stability is established. The discrete-time Markovian jump linear system (MJLS) approach for NCS is considered in [17]. Based on the Lyapunov method, an iterative linear matrix inequality (LMI) for the mode-dependent controller is proposed. However, only the stochastic stability is quaranteed.

In this paper, stochastic exponential mean square stability for longer random transmission delay with upper bound is considered. The sensor-to-controller (SC) delay $\tau_{\mathrm{sc}}\left(r_{t}\right)$ is modelled by a Markovian process $r_{t}$ while the controllerto-actuator (CA) delay $\bar{\tau}_{\mathrm{ca}}$ is held constant by the buffering technique. The sampled-data system approach is applied and a delay-dependent switching state-feedback controller is proposed. The resulting delay contains a random part $\tau_{\mathrm{sc}}\left(r_{t}\right)$ related to network transmission and a linear time-varying part, which is uncertain and bounded by the sampling interval. The switching controller monitors the SC random delay and synchronously switches with it. As a result, an MJLS with random delay is established. A delay-dependent stability condition for stochastic exponential mean square stability is derived by using a Lyapunov-Krasovskii functional. A simple switching controller design algorithm is proposed. All the results are presented in terms of linear matrix inequalities (LMI's). The proposed switching controller approach is experimentally validated using a 3 DoF robotic manipulator ViSHaRD3 [18]. An alternative non-switching approach by using a buffering approach rendering the SC transmission delay constant is considered. The experimental comparison with the non-switching counterpart indicates performance benefits for the proposed switching control approach.

The remainder of the paper is organized as follows: In section II, the sampled-data MJLS is introduced. The system contains a random delay and a switching state-feedback 


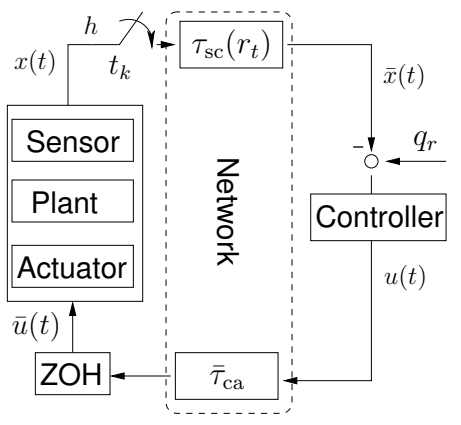

Fig. 1. Illustration of NCS over communication network, the transmission delay from sensor-to-controller $\tau_{\mathrm{sc}}\left(r_{t}\right)$ and from controller-to-actuator $\bar{\tau}_{\mathrm{ca}}$.

controller. In section III, an stochastic exponential mean square stability condition for MJLS is derived. Based on the stability condition, a switching controller design algorithm is proposed in section IV. The experimental validation and performance comparison are illustrated in section $\mathrm{V}$.

Notation. Throughout the paper we let $\lambda_{\max }(M)$ and $\lambda_{\min }(M)$ denote the maximal and the minimal eigenvalue of matrix $M . M^{T}$ and $\|M\|$ denote the transpose and induced Euclidean norm of matrix (or vector) $M$. Let $\left\|e\left(t_{f}\right)\right\|_{2}$ denote the $L_{2}$ norm of $e(t)$ on a given interval $\left[0 t_{f}\right]$, where $\left\|e\left(t_{f}\right)\right\|_{2}=\sqrt{\int_{0}^{t_{f}} e(t) e^{T}(t) d t}$. $\mathbb{E}$ stands for mathematical expectation and $\mathbf{P}$ for probability. Let $\left\{r_{t}, t \geq 0\right\}$ denote a Markov process governing the mode switching in the finite set $\mathcal{S}:=\{1, \ldots, N\}$ having the generator $\mathcal{A}=\left(\alpha_{i, j}\right)$, $i, j \in \mathcal{S}, \alpha_{i, j}>0, i \neq j, \alpha_{i, i}=-\sum_{i \neq j} \alpha_{i, j}$. Then the mode transition probability can be defined as

$$
\mathbf{P}_{i, j}\left(r_{t+\delta}=j \mid r_{t}=i\right)=e^{\mathcal{A} \delta} .
$$

\section{Problem Definition}

\section{A. NCS Model}

Consider an LTI system as plant

$$
\dot{x}(t)=A x(t)+B \bar{u}(t),
$$

where $x \in \mathbb{R}^{n}$ is the state and $\bar{u} \in \mathbb{R}^{m}$ is the control input; $A$ and $B$ are constant matrices with appropriate dimensions. The plant is interconnected by a controller over a communication network, see Fig. 1. The sensor and controller are periodically sampled with the sampling interval $h$.

We now consider the SC transmission delay as a Markovian time delay $\tau_{\mathrm{sc}}\left(r_{t}\right)$. The mode switching is governed by a Markov process $r_{t} \in \mathcal{S}$ taking values in the finite set $\mathcal{S}:=\{1, \ldots, N\}$. The switching rate from mode $i$ to mode $j$ is defined by $\alpha_{i, j}$. According to (1) and Fig. 1, the piecewise constant measurement from $\mathrm{SC}$ at the sampled time $t_{k}$ is given by

$$
\begin{aligned}
\bar{x}(t) & =x\left(t_{k}-\tau_{\mathrm{sc}}\left(r_{t}\right)\right)=x\left(t-\tau_{1}\left(t, r_{t}\right)\right), \\
\tau_{1}\left(t, r_{t}\right) & =t-t_{k}+\tau_{\mathrm{sc}}\left(r_{t}\right), \quad t_{k} \leq t<t_{k+1} .
\end{aligned}
$$

Assume a remote state-feedback controller which is able to monitor the SC delay, e.g. using the time-stamping technique, and synchronously switches the feedback gains with the SC

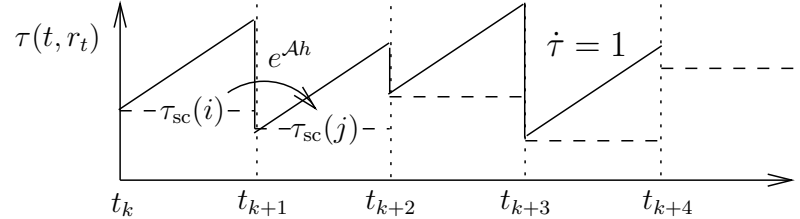

Fig. 2. The evolution of time delay $\tau\left(t, r_{t}\right)$ for certain sample path of $\tau_{\mathrm{sc}}\left(r_{t}\right)$.

delay $\tau_{\mathrm{sc}}\left(r_{t}\right)$. The control commands are fed back through the CA channel to the plant. Holding the CA delay constant $\bar{\tau}_{\text {ca }}$ by using buffering technique, we have the control law

$$
\bar{u}(t)=K\left(r_{t}\right) u\left(t-\bar{\tau}_{\mathrm{ca}}\right)=K\left(r_{t}\right) x\left(t-\tau_{1}\left(t, r_{t}\right)-\bar{\tau}_{\mathrm{ca}}\right) .
$$

Take (3) into system (1), the closed-loop system has the form

$$
\dot{x}(t)=A x(t)+B K\left(r_{t}\right) x\left(t-\tau\left(t, r_{t}\right)\right),
$$

where $\tau\left(t, r_{t}\right)=\tau_{1}\left(t, r_{t}\right)+\bar{\tau}_{\text {ca. }}$. System (4) is an MJLS with random delay $\tau\left(t, r_{t}\right)$.

\section{B. Time Delay Model}

The switching of transmission delays may result in the disorder of sampled sequence. In this paper we exclude the disordering in the sampled sequence, i.e. we assume

A1: $\mathbf{P}\left(\left|\tau_{\mathrm{sc}}\left(r_{t_{k+1}}\right)-\tau_{\mathrm{sc}}\left(r_{t_{k}}\right)\right| \geq h\right)=0$.

The assumption A1 restricts that the switching difference of consecutive delays is less than one sampling interval. This assumption is not unreasonable as the current transmission delay in the real communication networks is usually correlated to the previous delay. In single-path networks the assumption is automatically fulfilled.

The delay $\tau\left(t, r_{t}\right)$ contains a randomly piecewise constant part $\tau_{\mathrm{sc}}\left(r_{t}\right)$ related to the transmission delay and a timevarying part $t-t_{k}$ related to the inter-sampling effect as shown in Fig. 2. The time-varying part is bounded by a sampling interval, i.e. $t-t_{k} \leq h$, and has the derivative $\dot{\tau}=1$. We consider the time-varying part of delay as uncertain and bounded in the later analysis and let

$$
\tau\left(t, r_{t}\right)=h+\tau_{\mathrm{sc}}\left(r_{t}\right)+\bar{\tau}_{\mathrm{ca}} .
$$

Henceforth, we write $\tau\left(r_{t}\right)$ instead of $\tau\left(t, r_{t}\right)$ if no ambiguity occurs.

The associated upper and lower bounds of $\tau\left(r_{t}\right)$ are defined as

$$
\begin{aligned}
& \bar{\tau}=h+\max _{i \in \mathcal{S}}\left\{\tau_{\mathrm{sc}}(i)\right\}+\bar{\tau}_{\mathrm{ca}}, \\
& \underline{\tau}=h+\min _{i \in \mathcal{S}}\left\{\tau_{\mathrm{sc}}(i)\right\}+\bar{\tau}_{\mathrm{ca}} .
\end{aligned}
$$

Before the main result is introduced, the following definition and lemma have to be given.

Definition 1: System (4) is said to be stochastic exponential mean square stable if for any initial condition $x\left(t_{0}, r_{t_{0}}\right)$, there exist positive constants $b$, and $\rho$ such that for all $t \geq t_{0}$

$$
\mathbb{E}\left\{\|x(t)\|^{2} \mid x\left(t_{0}, r_{t_{0}}\right)\right\} \leq b\left\|x\left(t_{0}, r_{t_{0}}\right)\right\|^{2} e^{-\rho\left(t-t_{0}\right)} .
$$


Lemma 1: [19] Let $X$ and $Y$ be real constant matrices with appropriate dimensions. Then

$$
X^{T} Y+Y^{T} X \leq \varepsilon X^{T} X+\frac{1}{\varepsilon} Y^{T} Y
$$

holds for any $\varepsilon>0$.

\section{MAIN RESUlt}

In this section, a delay-dependent stability condition for an NCS with random delay is presented. The approach is derived by using the Lyapunov-Krasovskii functional and descriptor transformation. Introduce a $\gamma>0$, which is related to the convergence rate of $\mathbb{E}\|x(t)\|^{2}$ in (4) as discussed later, and consider a new variable $z(t)=e^{\gamma t} x(t)$. Substitute $z(t)$ into (4), it becomes

$$
\dot{z}(t)=(A+\gamma I) z(t)+e^{\gamma \tau\left(r_{t}\right)} B K\left(r_{t}\right) z\left(t-\tau\left(r_{t}\right)\right) .
$$

Note that

$$
z(t)-z\left(t-\tau\left(r_{t}\right)\right)=\int_{t-\tau\left(r_{t}\right)}^{t} \dot{z}(s) d s
$$

and substituting into (6) it gives

$$
\dot{z}(t)=\left(\hat{A}+\hat{A}_{1}\left(r_{t}\right)\right) z(t)-\hat{A}_{1}\left(r_{t}\right) \int_{t-\tau\left(r_{t}\right)}^{t} \dot{z}(s) d s,
$$

where $\hat{A}=A+\gamma I$ and $\hat{A}_{1}\left(r_{t}\right)=e^{\gamma \tau\left(r_{t}\right)} B K\left(r_{t}\right)$. Let $\xi^{T}(t)=\left[\begin{array}{ll}z^{T}(t) & \dot{z}^{T}(t)\end{array}\right]$, system (7) has the descriptor form

$$
E \dot{\xi}(t)=\left(\bar{A}+\bar{A}_{1}\left(r_{t}\right)\right) \xi(t)-\bar{A}_{2}\left(r_{t}\right) \int_{t-\tau\left(r_{t}\right)}^{t} \xi(s) d s,
$$

where

$$
\begin{aligned}
E & =\left[\begin{array}{ll}
I & 0 \\
0 & 0
\end{array}\right], \bar{A}=\left[\begin{array}{cc}
0 & I \\
\hat{A} & -I
\end{array}\right], \bar{A}_{1}\left(r_{t}\right)=\left[\begin{array}{cc}
0 & 0 \\
\hat{A}_{1}\left(r_{t}\right) & 0
\end{array}\right], \\
\bar{A}_{2}\left(r_{t}\right) & =\left[\begin{array}{cc}
0 & 0 \\
0 & \hat{A}_{1}\left(r_{t}\right)
\end{array}\right] .
\end{aligned}
$$

Theorem 1: For the closed-loop system (4) with a given decay rate $\gamma>0$ and positive scalars $n_{1}(i)$ and $n_{2}(i)$ if there exist matrices $Q>0$ and $X(i)=X^{T}(i)>0, i \in \mathcal{S}$ such that the following LMI holds

$$
\left[\begin{array}{cc}
\Psi_{1}(i) & \hat{\tau}(i) \Psi_{2}^{T}(i) \\
\hat{\tau}(i) \Psi_{2}(i) & -\hat{\tau}(i) Q
\end{array}\right]<0
$$

where

$$
\begin{aligned}
\hat{\tau}(i)= & \tau(i)+\frac{1}{2} \bar{\alpha}\left(\bar{\tau}^{2}-\underline{\tau}^{2}\right), \quad \bar{\alpha}=\max \left\{\left|\alpha_{i, i}\right|\right\}, \\
\Psi_{1}(i)= & \left(\bar{A}+\bar{A}_{1}(i)\right) X(i)+X^{T}(i)\left(\bar{A}+\bar{A}_{1}(i)\right)^{T} \\
& +\sum_{j=1}^{N_{\mathrm{sc}}} \alpha_{i, j} E X^{T}(j)+\tau(i) Q, \\
\Psi_{2}(i)= & \bar{A}_{2}(i) X(i),
\end{aligned}
$$

then the system is stochastic exponential mean square stable.

Proof: The state $\left\{\xi(t), r_{t}, t \geq 0\right\}$ depends on the history $\xi(t+\theta), \theta \in\left[-2 \tau\left(r_{t}\right), 0\right]$, which implies $\left\{\xi(t), r_{t}, t \geq 0\right\}$ is not a Markov process. According to [19], we modify our problem into a new Markov process $\left\{\Xi(t), r_{t}, t \geq 0\right\}$ having the values as the following

$$
\Xi(t)=\xi(s+t), s \in\left[t-2 \tau\left(r_{t}\right), t\right] .
$$

Define a set of positive definite matrices $P\left(r_{t}\right)=X^{-1}\left(r_{t}\right)$ and consider a Lyapunov candidate (10)

$$
\begin{aligned}
V\left(\Xi(t), r_{t}\right)= & V_{1}\left(\Xi(t), r_{t}\right)+V_{2}\left(\Xi(t), r_{t}\right) \\
& +V_{3}\left(\Xi(t), r_{t}\right),
\end{aligned}
$$

where

$$
\begin{aligned}
& V_{1}\left(\Xi(t), r_{t}\right)=\xi^{T}(t) E P\left(r_{t}\right) \xi(t), \\
& V_{2}\left(\Xi(t), r_{t}\right)=\int_{-\tau\left(r_{t}\right)}^{0} \int_{t+\theta}^{t} \xi^{T}(s) \bar{A}_{2}^{T}\left(r_{t}\right) Q^{-1} \bar{A}_{2}\left(r_{t}\right) \\
& \xi(s) d s d \theta, \\
& V_{3}\left(\Xi(t), r_{t}\right)=\bar{\alpha} \int_{-\bar{\tau}}^{-\underline{\tau}} \int_{t+\theta}^{t} \xi^{T}(s) \bar{A}_{2}^{T}\left(r_{t}\right) Q^{-1} \bar{A}_{2}\left(r_{t}\right) \xi(s) \\
&(s-t-\theta) d s d \theta .
\end{aligned}
$$

Suppose $r_{t}=i \in \mathcal{S}$ and let $\mathcal{L}(\cdot)$ be the infinitesimal generator of $\left\{\Xi(t), r_{t}, t \geq 0\right\}$; then

$$
\begin{aligned}
& \mathcal{L} V_{1}\left(\Xi(t), r_{t}\right)=\xi^{T}(t)\left[\left(\bar{A}+\bar{A}_{1}\left(r_{t}\right)\right)^{T} P\left(r_{t}\right)\right. \\
& \left.+P^{T}\left(r_{t}\right)\left(\bar{A}+\bar{A}_{1}\left(r_{t}\right)\right)+\sum_{j=1}^{N} \alpha_{i, j} E P(j)\right] \xi(t) \\
& -2 \xi^{T}(t) P^{T}\left(r_{t}\right) \bar{A}_{2}\left(r_{t}\right) \int_{t-\tau\left(r_{t}\right)}^{t} \xi(s) d s .
\end{aligned}
$$

According to Lemma $1, \mathcal{L} V_{1}\left(\Xi(t), r_{t}\right)$ becomes

$$
\begin{aligned}
& \mathcal{L} V_{1}\left(\Xi(t), r_{t}\right) \leq \xi^{T}(t)\left[\left(\bar{A}+\bar{A}_{1}\left(r_{t}\right)\right)^{T} P\left(r_{t}\right)\right. \\
& \left.+P^{T}\left(r_{t}\right)\left(\bar{A}+\bar{A}_{1}\left(r_{t}\right)\right)+\sum_{j=1}^{N} \alpha_{i, j} E P(j)\right] \xi(t) \\
& +\tau\left(r_{t}\right) \xi^{T}(t) P^{T}\left(r_{t}\right) Q P\left(r_{t}\right) \xi(t) \\
& +\int_{t-\tau\left(r_{t}\right)}^{t} \xi^{T}(s) \bar{A}_{2}^{T}\left(r_{t}\right) Q^{-1} \bar{A}_{2}\left(r_{t}\right) \xi(s) d s .
\end{aligned}
$$

Similarly,

$$
\begin{aligned}
& \mathcal{L} V_{2}\left(\Xi(t), r_{t}\right) \\
& \leq \tau\left(r_{t}\right) \xi^{T}(t) \bar{A}_{2}^{T}\left(r_{t}\right) Q^{-1} \bar{A}_{2}\left(r_{t}\right) \xi(t) \\
& -\int_{t-\tau\left(r_{t}\right)}^{t} \xi^{T}(s) \bar{A}_{2}^{T}\left(r_{t}\right) Q^{-1} \bar{A}_{2}\left(r_{t}\right) \xi(s) d s \\
& +\bar{\alpha} \int_{-\bar{\tau}}^{-\underline{\tau}} \int_{t+\theta}^{t} \xi^{T}(s) \bar{A}_{2}^{T}\left(r_{t}\right) Q^{-1} \bar{A}_{2}\left(r_{t}\right) \xi(s) d s d \theta . \\
& \mathcal{L} V_{3}\left(\Xi(t), r_{t}\right) \\
& =\frac{1}{2} \bar{\alpha}\left(\bar{\tau}^{2}-\underline{\tau}^{2}\right) \xi^{T}(t) \bar{A}_{2}^{T}\left(r_{t}\right) Q^{-1} \bar{A}_{2}\left(r_{t}\right) \xi(t) \\
& -\bar{\alpha} \int_{-\bar{\tau}}^{-\tau} \int_{t+\theta}^{t} \xi^{T}(s) \bar{A}_{2}^{T}\left(r_{t}\right) Q^{-1} \bar{A}_{2}\left(r_{t}\right) \xi(s) d s d \theta .
\end{aligned}
$$


Combining (11)-(13), it results in

$$
\begin{aligned}
& \mathcal{L} V\left(\Xi(t), r_{t}\right) \leq \xi^{T}(t)\left[\left(\bar{A}+\bar{A}_{1}\left(r_{t}\right)\right)^{T} P\left(r_{t}\right)\right. \\
& \left.+P^{T}\left(r_{t}\right)\left(\bar{A}+\bar{A}_{1}\left(r_{t}\right)\right)+\sum_{j=1}^{N} \alpha_{i, j} E P(j)\right] \xi(t) \\
& +\tau\left(r_{t}\right) \xi^{T}(t) P^{T}\left(r_{t}\right) Q P\left(r_{t}\right) \xi(t) \\
& +\tau\left(r_{t}\right) \xi^{T}(t) \bar{A}_{2}^{T}\left(r_{t}\right) Q^{-1} \bar{A}_{2}\left(r_{t}\right) \xi(t) \\
& +\frac{1}{2} \bar{\alpha}\left(\bar{\tau}^{2}-\underline{\tau}^{2}\right) \xi^{T}(t) \bar{A}_{2}^{T}\left(r_{t}\right) Q^{-1} \bar{A}_{2}\left(r_{t}\right) \xi(t) \\
& =\xi^{T}(t) \Theta\left(r_{t}\right) \xi(t) .
\end{aligned}
$$

Pre- and post-multiply $\Theta\left(r_{t}\right)$ by $X^{T}\left(r_{t}\right)$ and $X\left(r_{t}\right)$, it gives

$$
\begin{aligned}
& \left(\bar{A}+\bar{A}_{1}\left(r_{t}\right)\right) X\left(r_{t}\right)+X^{T}\left(r_{t}\right)\left(\bar{A}+\bar{A}_{1}\left(r_{t}\right)\right)^{T} \\
& +\hat{\tau}\left(r_{t}\right) X^{T}\left(r_{t}\right) \bar{A}_{2}^{T}\left(r_{t}\right) Q^{-1} \bar{A}_{2}\left(r_{t}\right) X\left(r_{t}\right) \\
& +\sum_{j=1}^{N} \alpha_{i, j} E X^{T}(j)+\tau\left(r_{t}\right) Q<0 .
\end{aligned}
$$

Applying Schur complement to (15) it results in (9).

Since $\max _{\theta \in[-2 \tau, 0]}\{\|\xi(t+\theta)\|\} \leq \varphi\|\xi(t)\|$ for some $\varphi>0$ [20]. Therefore, it has

$$
\begin{aligned}
V\left(\Xi(t), r_{t}\right) & \leq\left[\lambda_{\max }\left(E P\left(r_{t}\right)\right)+\varphi \lambda_{\max }(R)\right]\|\xi(t)\|^{2} \\
& \leq \Lambda_{\max }\left(r_{t}\right)\|\xi(t)\|^{2},
\end{aligned}
$$

where

$$
R=\bar{A}_{2}^{T}\left(r_{t}\right) Q^{-1} \bar{A}_{2}\left(r_{t}\right), \quad \varphi=\frac{1}{2} \bar{\tau}^{2}+\frac{1}{6}\left(\bar{\tau}^{3}-\underline{\tau}^{3}\right) \bar{\alpha} .
$$

and

$$
\Lambda_{\max }\left(r_{t}\right)=\lambda_{\max }\left(E P\left(r_{t}\right)\right)+\varphi \lambda_{\max }(R) .
$$

Combining with (14), it becomes

$$
\frac{\mathcal{L} V\left(\Xi(t), r_{t}\right)}{V\left(\Xi(t), r_{t}\right)} \leq-\min _{r_{t} \in \mathcal{S}}\left\{\frac{\lambda_{\min }\left(-\Theta\left(r_{t}\right)\right)}{\Lambda_{\max }\left(r_{t}\right)}\right\} \triangleq-\rho_{0}
$$

and yields in

$$
\mathbb{E} \mathcal{L} V\left(\Xi(t), r_{t}\right) \leq-\rho_{0} \mathbb{E} V\left(\Xi(t), r_{t}\right) .
$$

Applying Dynkin's formula into (16), we have

$$
\begin{aligned}
& \mathbb{E} V\left(\Xi(t), r_{t}\right)-\mathbb{E} V\left(\Xi(0), r_{0}\right) \\
& =\mathbb{E}\left[\int_{0}^{t} \mathcal{L} V\left(\Xi(s), r_{s}\right) d s\right] \\
& \leq-\rho_{0} \int_{0}^{t} \mathbb{E} \mathcal{L} V\left(\Xi(s), r_{s}\right) d s .
\end{aligned}
$$

Using the Gronwall-Bellman lemma, (17) results in

$$
\mathbb{E} V\left(\Xi, r_{t}\right) \leq e^{-\rho_{0} t} \mathbb{E} V\left(\Xi(0), r_{0}\right) .
$$

Since

$$
\begin{aligned}
V\left(\Xi(t), r_{t}\right) & \geq\left[\lambda_{\min }\left(E P\left(r_{t}\right)\right)+\varphi \lambda_{\min }(R)\right]\|\xi(t)\|^{2} \\
& =\Lambda_{\min }\left(r_{t}\right)\|\xi(t)\|^{2}
\end{aligned}
$$

it becomes

$$
\mathbb{E}\|\xi(t)\|^{2} \leq e^{-\rho_{0} t} \frac{\mathbb{E} V\left(\Xi(0), r_{0}\right)}{\min _{r_{t} \in \mathcal{S}}\left\{\Lambda_{\min }\left(r_{t}\right)\right\}} .
$$

Equation (18) implies stochastic exponential mean square stability and completes the proof.

Remark 1: The delay $\tau\left(r_{t}\right)$ contains the transmission delay and uncertain time-varying component bounded by a sampling interval, see (5). Accordingly, the transmission delay as well as the sampling interval are conjointly treated by a single stability condition in Theorem 1 . The solution of Theorem 1 indicates the trade-off between transmission delays $\tau_{\mathrm{sc}}\left(r_{t}\right)+\bar{\tau}_{\mathrm{ca}}$ and the sampling interval $h$ for which the stochastic exponential mean square stability is guaranteed.

Remark 2: In case of constant transmission delay, i.e. $\tau_{\mathrm{sc}}\left(r_{t}\right)=\tau_{\mathrm{sc}}$ and $\alpha_{i, j}=0$, Theorem 1 is straightforward applicable to systems with constant delay. For random SC and CA transmission delays, the extended results of the same author can be found in [21].

Remark 3: It is noted that $\mathbb{E}|| \xi(t)\left\|^{2} \geq \mathbb{E}\right\| z(t) \|^{2}$ and $z(t)=e^{\gamma t} x(t)$. Therefore, the inequality (18) can be rewritten as

$$
\mathbb{E}|| x(t) \|^{2} \leq e^{-\left(\rho_{0}+2 \gamma\right) t} \frac{\mathbb{E} V\left(\Xi(0), r_{0}\right)}{\min _{r_{t} \in \mathcal{S}}\left\{\Lambda_{\min }\left(r_{t}\right)\right\}} .
$$

The given $\gamma$ in Theorem 1 ensures the smallest decay rate of trajectory $\mathbb{E}\|x(t)\|^{2}$.

\section{Controller Design}

The difficulty in solving switching feedback gain $K(i)$ in the matrix inequality (9) involves the nonlinear terms, i.e. $\bar{A}_{1}(i) X(i)$ in $\Psi_{1}(i)$ and $\bar{A}_{2}(i) X(i)$ in $\Psi_{2}(i)$, and cannot be considered as an LMI problem. However, by introducing special settings of $X(i)$ the nonlinear terms can be eliminated and the LMI problem is recovered.

Theorem 2: For given positive scalars $n_{1}(i), n_{2}(i)$ and decay rate $\gamma>0$, if there exist matrices $Q>0$, $X_{11}(i)=X_{11}^{T}(i)>0$ satisfying

$$
X(i)=\left[\begin{array}{cc}
X_{11}(i) & 0 \\
-n_{1}(i) X_{11}(i) & n_{2}(i) X_{11}(i)
\end{array}\right]
$$

and matrices $Y(i)$ satisfying

$$
\bar{Y}(i)=\left[\begin{array}{ll}
Y(i) & 0
\end{array}\right],
$$

$i \in \mathcal{S}$ such that

$$
\left[\begin{array}{cc}
\hat{\Psi}_{1}(i) & \hat{\tau}(i) \hat{\Psi}_{2}^{T}(i) \\
\hat{\tau}(i) \hat{\Psi}_{2}(i) & -\hat{\tau}(i) Q
\end{array}\right]<0,
$$

where

$$
\begin{aligned}
\hat{\tau}(i)= & \tau(i)+\frac{1}{2} \bar{\alpha}\left(\bar{\tau}^{2}-\underline{\tau}^{2}\right), \quad \bar{\alpha}=\max \left\{\left|\alpha_{i, i}\right|\right\}, \\
\hat{\Psi}_{1}(i)= & \bar{A} X(i)+\bar{B} \bar{Y}(i)+(\bar{A} X(i)+\bar{B} \bar{Y}(i))^{T} \\
& +\sum_{j=1}^{N_{\mathrm{sc}}} \alpha_{i, j} E X^{T}(j)+\tau(i) Q, \\
\bar{B}^{T}= & {\left[\begin{array}{ll}
0 & B^{T}
\end{array}\right], }
\end{aligned}
$$




$$
\hat{\Psi}_{2}(i)=\left[\begin{array}{cc}
0 & 0 \\
-n_{1}(i) e^{\gamma \tau(i)} B Y(i) & n_{2}(i) e^{\gamma \tau(i)} B Y(i)
\end{array}\right],
$$

holds, then the closed-loop system (4) is stochastic exponential mean square stable with the feedback gain

$$
K(i)=Y(i) X_{11}^{-1} .
$$

Proof: According to Theorem 1, the switching controller (3) stabilizes the closed-loop system (4) if the inequality (9) is satisfied. Subsitute (19), (20) and $\bar{B}^{T}=\left[\begin{array}{ll}0 & B^{T}\end{array}\right]$ into (9) and let $Y(i)=K(i) X_{11}(i)$. The nonlinear terms in (9) are eliminated and the LMI (21) is derived.

\section{EXPERIMENTAL VALIDATION}

In order to validate the proposed switching control approach, experiments of the position control for a $3 \mathrm{DoF}$ robotic manipulator ViSHaRD3 [18] are conducted. The device is equipped with a fixed end-effector and three revolute joints as shown in Fig. 3. Each joint is actuated by a Maxon RE40 DC motor coupled with a harmonic drive gear (gear ratio 1:100). The DC-motor current, resulting in torque, is provided by the PWM amplifier operated under current control. The reference signal is given by voltage from D/A converter and is an output of the I/O board. The ViSHaRD3 device is connected to a PC running RT Linux. The control loop and the communication network, i.e. the transmission delay, are implemented in MATLAB/SIMULINK blocksets. Standalone realtime code is generated directly from the SIMULINK models.

\section{A. Experimental System Model}

Due to the requirement of the proposed approach, the ViSHaRD3 device is linearized by computed torque feedforward approach [22]. Combined with friction compensation, the linearized ViSHaRD3 system is decoupled into three systems

$$
\frac{d}{d t}\left[\begin{array}{l}
q_{i} \\
\dot{q}_{i}
\end{array}\right]=\left[\begin{array}{cc}
0 & 1 \\
1 & -50
\end{array}\right]\left[\begin{array}{l}
q_{i} \\
\dot{q}_{i}
\end{array}\right]+\left[\begin{array}{l}
0 \\
1
\end{array}\right] u_{i}
$$

$i=1,2$ for joint 1,2

$$
\frac{d}{d t}\left[\begin{array}{l}
q_{3} \\
\dot{q}_{3}
\end{array}\right]=\left[\begin{array}{cc}
0 & 1 \\
1 & -40
\end{array}\right]\left[\begin{array}{l}
q_{3} \\
\dot{q}_{3}
\end{array}\right]+\left[\begin{array}{l}
0 \\
1
\end{array}\right] u_{3}
$$

for joint 3, where $q=\left[q_{1}, q_{2}, q_{3}\right]^{T}$.

The joint vector $q$ of ViSHaRD3 is fed to the remote controller through a communication network having the SC delay $\tau_{\mathrm{sc}}\left(r_{t}\right) \in\{1,3,7\} \mathrm{ms}$ and the transition rate

$$
\mathcal{A}=\left[\begin{array}{ccc}
-3 & 2 & 1 \\
1 & -3 & 2 \\
3 & 1 & -4
\end{array}\right]
$$

The ViSHaRD3 system is stabilized by a set of PD controllers, which are synchronously switched with the SC delay. Combine the switching PD controller into (23) and (24), it yields

$$
\dot{q}(t)=A_{i} q(t)+\bar{K}\left(r_{t}\right) q\left(t-\tau\left(r_{t}\right)\right),
$$

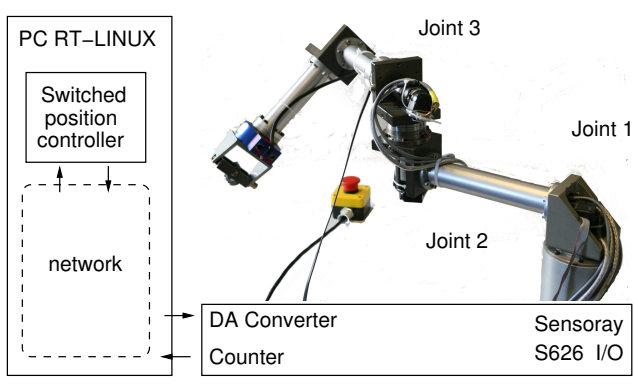

Fig. 3. Experimental 3 DoF ViSHaRD3 system.

where $i=1,2,3$ and

$$
\begin{aligned}
A_{1}=A_{2} & =\left[\begin{array}{cc}
0 & 1 \\
1 & -50
\end{array}\right], A_{3}=\left[\begin{array}{cc}
0 & 1 \\
1 & -40
\end{array}\right], \\
\bar{K} & =\left[\begin{array}{cc}
0 & 0 \\
-K_{P}\left(r_{t}\right) & -K_{D}\left(r_{t}\right)
\end{array}\right] .
\end{aligned}
$$

The PD gains in (25) are computed by (21) using the ralmip toolbox [23] in MATLAB. With the sampling interval $h=10 \mathrm{~ms}, \quad \mathrm{CA}$ transmission delay $\bar{\tau}_{\mathrm{ca}}=1 \mathrm{~ms}$, the resulting delay in (5) has the values $\tau(1)=12 \mathrm{~ms}, \tau(2)=14 \mathrm{~ms}, \tau(3)=18 \mathrm{~ms}$. Set the decay rate to be $\gamma=2.5$. Solve the LMI (21) in Theorem 2 by using brute-force search of $n_{1}(i), n_{2}(i)$, where $i \in \mathcal{S}:=\{1,2,3\}$. The feasible PD gains are computed with $n_{1}(1)=n_{2}(1)=1.6 \times 10^{3}, \quad n_{1}(2)=n_{2}(2)=3.6 \times 10^{3}$ and $n_{1}(3)=n_{2}(3)=1.7 \times 10^{6}$ and summarized in Table I.

TABLE I

The feasible switching PD controller for ViSHaRD3 device

\begin{tabular}{|l|c|c|}
\hline & Joint $1 / 2$ & Joint 3 \\
\hline$\tau(1)=12 \mathrm{~ms}$ & $K_{P}(1)=259.65$ & $K_{P}(1)=208.95$ \\
$\tau_{\text {sc }}(1)=1 \mathrm{~ms}$ & $K_{D}(1)=5.19$ & $K_{D}(1)=5.21$ \\
\hline$\tau(2)=14 \mathrm{~ms}$ & $K_{P}(2)=96.54$ & $K_{P}(2)=77.39$ \\
$\tau_{\mathrm{sc}}(2)=3 \mathrm{~ms}$ & $K_{D}(2)=1.93$ & $K_{D}(2)=1.93$ \\
\hline$\tau(3)=18 \mathrm{~ms}$ & $K_{P}(3)=77.21$ & $K_{P}(3)=23.26$ \\
$\tau_{\mathrm{sc}}(3)=7 \mathrm{~ms}$ & $K_{D}(3)=1.55$ & $K_{D}(3)=1.21$ \\
\hline
\end{tabular}

\section{B. Experiments}

The initial joint vector of ViSHaRD3 is set to $q^{T}\left(t_{0}\right)=\left[\begin{array}{lll}0 & 0 & -0.5 \pi\end{array}\right] \mathrm{rad}$ and $\dot{q}^{T}\left(t_{0}\right)=\left[\begin{array}{lll}0 & 0 & 0 \pi\end{array}\right] \mathrm{rad} / \mathrm{s} . \quad \mathrm{A}$ sinusoidal function, which has the amplitude 0.2 and frequency $0.5 \mathrm{rad} / \mathrm{s}$, serves as position reference $q_{r}$ to the system. The experiments are run 10 times with random initial distribution probabilities of SC delay. One sample path of the SC delay is shown in Fig 4 (a). Two approaches are investigated. In the proposed switching control approach, the delay is monitored using the time-stamping technique and the remote controller is synchronously switched with the SC delay. The second approach holds the SC delay constant by using the buffering technique at the controller side, i.e. the controller is designed with the higher delay $\tau_{\mathrm{sc}}(3)$. For comparison, the evolutions of normalized mean control error ${ }^{2}$ $\bar{e}(t)$ are shown in Fig. 4 (b). It is observed that the normalized mean control errors of the proposed approach (solid line) are

\footnotetext{
${ }^{2}$ The normalized control error is defined as $\bar{e}(t)=\frac{q(t)-q_{r}\left(t-\tau\left(r_{t}\right)\right.}{\max \left\{\left\|q_{r}(t)\right\|\right\}}$.
} 


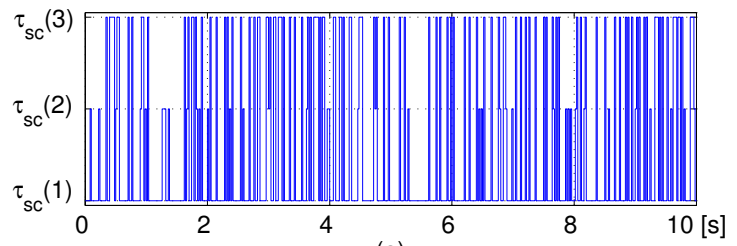

(a)
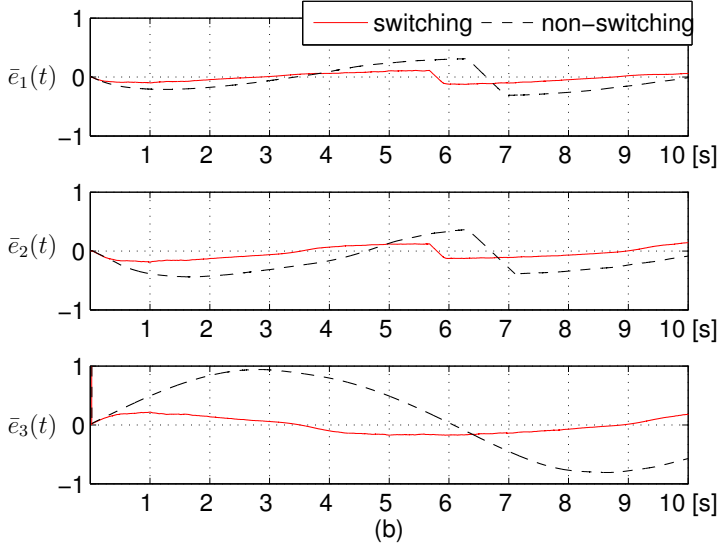

Fig. 4. The sample path of Markovian delay for the experiment (a) and mean control error evolutions with switching controller (solid line) and nonswitching controller (dashed line) (b)

in general smaller than the non-switching approach (dashed line). The $L_{2}$ norm of normalized mean control error over the experimental time horizon $\left[0 t_{f}\right], t_{f}=10 \mathrm{~s}$, is measured to be $\left\|\bar{e}\left(t_{f}\right)\right\|_{2}=1.42$ for the proposed switching control approach and $\left\|\bar{e}\left(t_{f}\right)\right\|_{2}=3.92$ for non-switching approach. Surprisingly, the switching control approach has superior performance benefits over the non-switching counterpart even when the total switching difference of delay is small, as in this case only $6 \mathrm{~ms}$. In case of larger switching delay difference, the performance benefit is likely to be more obvious. The experiment shows the proposed approach is very promising for the NCS applications.

Open questions that will be addressed in the future research concerns the control performance optimization and the packet dropout.

\section{CONCLUSIONS}

Aiming at the networked control system (NCS) with random transmission delay this paper concerns a novel control approach based on Markovian jump linear systems with random delay and gives a sufficient stability condition and controller design algorithm in terms of linear matrix inequalities (LMI's). Stochastic exponential mean square stability is guaranteed for longer random transmission delay by using the Lyapunov-Krasovskii functional. A switching statefeedback controller is proposed and validated by a 3 DoF robotic manipulator ViSHaRD3. The experiment demonstrates the superior performance benefit of the proposed switching controller over the non-switching counterpart. This confirms the proposed control approach is very promising for NCS applications.

\section{ACKNOWLEDGMENTS}

This work was supported in part by the German Research Foundation (DFG) within the Priority Program SPP 1305 "Control Theory of Digitally Networked Dynamical Systems".

\section{REFERENCES}

[1] P. Seiler, Coordinated control of unmanned aerial vehicles. $\mathrm{PhD}$ thesis, Univ. California, Berkeley, 2001

[2] R. Daoud, H. Amer, H. Elsayed, and Y. Sallez, "Ethernet-based car control network," in IEEE CCECE/CCGEI, (Ottawa), 2006.

[3] S. Hirche, Haptic Telepresence in Packet Switched Communication Networks. PhD thesis, Technische Universität München, Institute of automatic control engineering, 2005.

[4] J. Baillieul and P. Antsaklis, "Control and communication challenges in networked real-time systems," Proceedings of the IEEE, vol. 95, pp. 9-28, Jan. 2007.

[5] K. Gu, V. L. Kharitonov, and J. Chen, Stability of Time-Delay Systems. Boston: Birkhäuser, 2003.

[6] A. Ray and Y. Galevi, "Integrated communication and control systems: Part II-design considerations," Journal of Dynamic Systems, Measurements, and Control, vol. 110, pp. 374-381, 1988.

[7] W. Zhang, M. S. Branicky, and S. M. Philips, "Stability of network control systems," IEEE Control Systems Magazine, vol. 21, pp. 84-99, February 2001.

[8] H. Lin, G. Zhai, and P. J. Antsaklis, "Robust stability and disturbance attenuation analysis of a class of networked control systems," in 42nd IEEE Conference on Decision and Control, (Maui), pp. 1182-1187, 2003.

[9] E. Fridman, A. Seuret, and J.-P. Richard, "Robust sampled-data stabilization of linear systems: an input delay approach," Automatica, vol. 40, pp. 1441-1446, 2004.

[10] E. Witrant, C. C. de Wit, D. Georges, and M. Alamir, "Remote stabilization via communication networks with a distributed control law," IEEE Transactions on Automatic Control, vol. 52, no. 8, pp. 1480$1485,2007$.

[11] V. L. Kharitonov, "Robust stability analysis of time delay systems: A survey," Annual Reviews in Control, vol. 23, pp. 185-196, 1999.

[12] J.-P. Richard, "Time-delay systems: an overview of some recent advances and open problems," Automatica, vol. 39, pp. 1667-1694, 2003

[13] J. Nilsson, Real-time control systems with delay. PhD thesis, Lund Institute of Technology, 1998.

[14] L. Xiao, A. Hassibi, and J. P. How, "Control with random communication delays via a discrete-time jump system approach," in Proceedings of the American Control Conference, (Chicago, Illinois), June 2000.

[15] L. Montestruque and P. Antsaklis, "Stability of model-based networked control systems with time-varying transmission times," IEEE Transactions on Automatic Control, vol. 49, no. 9, pp. 1562-1572, 2004.

[16] F. Yang, Z. Wang, Y. S. Hung, and M. Gani, " $H_{\infty}$ control for networked control systems with random communication delays," IEEE Transactions on Automatics Control, vol. 51, pp. 511-518, Mar. 2006.

[17] L. Zhang, Y. Shi, T. Chen, and B. Huang, "A new method for stabilization of networked control systems with random delays," IEEE Transactions on Automatic Control, vol. 20, no. 8, pp. 1177-1181, 2005.

[18] M. Ueberle, Design, Control, and Evaluation of a Family of Kinematic Haptic Interfaces. $\mathrm{PhD}$ thesis, Technische Universität München, Institute of automatic control engineering, 2006.

[19] E.-K. Boukas and Z.-K. Liu, Deterministic and Stochastic Time Delay Systems. Boston: Birkhäuser, 2002.

[20] M. S. Mahmoud and N. F. Al-Muthairi, "Design of robust controller for time-delay systems," IEEE Transactions on Automatic Control, vol. 39, pp. 995-999, 1984.

[21] C.-C. Chen, S. Hirche, and M. Buss, "Sampled-data networked control systems with random time delay," in IFAC 2008, (Seoul), July 2008.

[22] L. Sciavicco and B. Siciliano, Modelling and Control of Robot Manipulators. London: Springer, 2 ed., 2001.

[23] J. Löfberg, "Yalmip : A toolbox for modeling and optimization in MATLAB," in Proceedings of the CACSD Conference, (Taipei, Taiwan), 2004 